\title{
X-ray diffraction study of AIN/AIGaN short period superlattices
}

\author{
A. Chandolu, S. Nikishin, ${ }^{\text {a) }}$ M. Holtz, and H. Temkin \\ Nano Tech Center, Texas Tech University, Lubbock, Texas 79409, USA
}

(Received 23 August 2007; accepted 12 October 2007; published online 10 December 2007)

\begin{abstract}
Short period superlattices of $\mathrm{AlN} / \mathrm{Al}_{0.08} \mathrm{Ga}_{0.92} \mathrm{~N}$ with the average $\mathrm{AlN}$ content over $60 \%$ have been investigated by high resolution X-ray diffraction. The $a$ and $c$ lattice constants verify these structures to be strain relaxed. Monolayer-level interface roughness, caused by the presence of threading dislocations and step-flow growth mode, is simulated and directly compared with the zeroth and \pm 1 satellite peak positions of the rocking curves. It was found that the observed $\mathrm{x}$-ray diffraction data can be adequately described by considering primarily the presence of screw dislocations and step-flow growth mode. (C) 2007 American Institute of Physics. [DOI: 10.1063/1.2821358]
\end{abstract}

\section{INTRODUCTION}

Unique optical and electrical properties of short period superlattices (SPSLs) of AlGaN/AlN make them valuable in optoelectronic devices operating in the ultraviolet. ${ }^{1}$ Growth and structural properties of SPSLs, along with AlGaN alloys and multiple quantum well structures, have been the subject of considerable recent interest. ${ }^{1-7}$ This paper examines $\mathrm{AlGaN} / \mathrm{AlN}$ SPSLs with periods ranging between five and ten monolayers (MLs) by high resolution x-ray diffraction (HRXRD). Detailed simulations of measured positions of the zeroth and \pm 1 satellite peaks of SPSLs allow us to determine their average compositions, periods, and barrier and well thicknesses. We model interfacial roughness at both the wellbarrier and barrier-well interfaces taking into account high density of the threading dislocations (TDs) in AlGaN alloys. In addition, we discuss how TDs (screw, mixed, and edge) influence the long range $2 \theta-\omega$ scan x-ray measurements.

\section{SAMPLE PREPARATION}

SPSLs were grown by gas source molecular beam epitaxy (GSMBE) with ammonia on (0001) sapphire substrates. The typical structure consists of $\sim 40 \mathrm{~nm}$ thick buffer layer of $\mathrm{AlN}$, followed by a $\sim 100 \mathrm{~nm}$ thick $\mathrm{Al}_{0.4} \mathrm{Ga}_{0.6} \mathrm{~N}$ layer grown in two dimensional (2D) growth mode to obtain a flat surface, and the SPSL containing 400 pairs of $\mathrm{AlN} / \mathrm{Al}_{\mathrm{x}} \mathrm{Ga}_{1-x} \mathrm{~N}(0.07<x<0.9)$, all grown in the $2 \mathrm{D}$ growth mode. The growth mode was monitored in situ using reflection high energy electron diffraction (RHEED). The complete growth details have been described elsewhere. ${ }^{1,5,8-11}$ Using atomic force microscope (AFM) the root mean squared (rms) surface roughness of $\sim 1 \mathrm{~nm}$ was measured for all 2D grown samples. ${ }^{10}$ Wells with the nominal thicknesses of $\sim 0.52$ and $\sim 0.78 \mathrm{~nm}$ were sandwiched between barrier layers with thickness nominally varying from $\sim 0.75$ to $\sim 1.75 \mathrm{~nm}$, resulting in periods ranging from 1.27 to $2.53 \mathrm{~nm}$. Note that the measured ML thickness for AlN is $0.249 \mathrm{~nm}$ and that for $\mathrm{Al}_{0.08} \mathrm{Ga}_{0.92} \mathrm{~N}$, determined by Vegard's law, is $0.258 \mathrm{~nm}$.

${ }^{a)}$ Electronic mail: sergey.a.nikishin@ttu.edu

\section{EXPERIMENTAL RESULTS AND DISCUSSIONS}

HRXRD studies were performed using a high-resolution diffractometer in double crystal alignment. A four-bounce Bartels Ge-220 monochromator was used for selecting $\mathrm{Cu} K \alpha_{1}$ radiation. We were able to measure the first and the second order satellite peaks, but the intensity of second order peaks was too weak to be considered for simulations. The intensity of the first order satellite peaks was sufficient to quantitatively analyze the x-ray data. In order to observe the intense second order superlattice peaks the monochromator was replaced with a hybrid mirror. A long range $2 \theta-\omega$ scan of the (0002) reflection is shown in Fig. 1 for a typical SPSL. Although the angular positions of x-ray reflections are well defined, this setup has the unfortunate by-product of causing additional broadening of all peaks due to the unresolved $\mathrm{Cu} K \alpha_{1}$ and $K \alpha_{2}$ peaks. These broadened XRD peaks are unsuitable for the detailed simulations carried out here. The $2 \theta-\omega$ scans of the symmetric (0002) reflections were employed to determine the SPSL period and the average AlN content $(y)$, and the respective thicknesses of the well and barrier layers. The $2 \theta$ scans of the symmetric (0002) and asymmetric $(11 \overline{2} 4)$ reflections were used to accurately determine the strained lattice parameters, $a^{*}$ and $c^{*}$, following the method described by Fewster et al. ${ }^{12}$ A three-bounce Ge-220 analyzer crystal was used for these high-resolution measurements. A precise knowledge of both SPSL lattice parameters is important for accurately determining the stress, degree of relaxation, and the average composition. The full width at half maximum (FWHM) of $\omega$ scans for the symmetric (0002) reflection was used to estimate the pure screw and/or screw

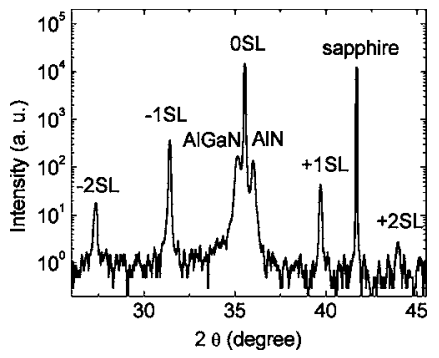

FIG. 1. A long range $2 \theta-\omega$ scan of the (0002) reflection obtained using a hybrid x-ray mirror. 


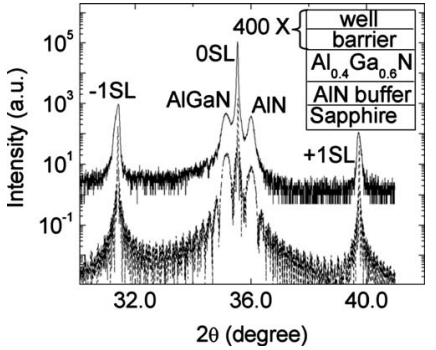

FIG. 2. A long range $2 \theta-\omega$ scan of (0002) reflections for a typical SPSL sample schematically illustrated in the inset. Solid line: data; dashed line: simulations.

component of mixed dislocation densities. ${ }^{13,14}$ The FWHM dependences of $(10 \overline{1} 5),(10 \overline{1} 3),(10 \overline{1} 1),(11 \overline{2} 4)$, and $(21 \overline{3} 3)$ $\Phi$ and $\omega$ scans on the inclination angle $\phi$ were used to estimate the pure edge and/or edge component of mixed dislocation densities. ${ }^{15,16}$ Note that the mixed TDs cause broadening in both symmetric and asymmetric X-ray peaks. ${ }^{17}$

The solid curve in Fig. 2 shows a long range $2 \theta-\omega$ scan of the (0002) reflection for a typical sample (schematically illustrated in the inset). Based on the growth conditions for this sample we expect the average AlN content $y=0.685$, SPSL period of $2.268 \mathrm{~nm}, \mathrm{Al}_{0.08} \mathrm{Ga}_{0.92} \mathrm{~N}$ well thickness of $0.774 \mathrm{~nm}$ (three MLs), and AlN barrier thickness of $1.494 \mathrm{~nm}$ (six MLs). Individual peaks corresponding to the AlN buffer layer, $\mathrm{Al}_{0.4} \mathrm{Ga}_{0.6} \mathrm{~N}$ layer, and the zeroth and \pm 1 satellites of the SPSL are well defined in Fig. 2. The average SPSL composition of $y=0.68$ is determined from the $2 \theta$ position of the zeroth peak and it is in excellent agreement with the expected value. From the position of the zeroth and \pm 1 satellite peaks the average period of the SPSL was determined to be $2.236 \mathrm{~nm}$. Using the experimentally determined period and assuming well composition of $\mathrm{Al}_{0.08} \mathrm{Ga}_{0.92} \mathrm{~N}$ and pure AlN barriers, the well and the barrier thicknesses are found to be 0.808 and $1.428 \mathrm{~nm}$, respectively.

Simulations based on the experimentally determined SPSL parameters yield an excellent fit to the experimental data as shown by the dashed curve in Fig. 2. However, this fit uses barrier and well thickness values which do not correspond to integer (9) ML multiples. The period is smaller, by $-0.032 \mathrm{~nm}$ than the expected multiple ML value, and the well and barrier thicknesses deviate from expected values by +0.034 and $-0.066 \mathrm{~nm}$, respectively. A simulation using nominal three ML wells and six ML barriers, namely, the ideal SPSL (ISPSL), does not produce a good fit to the data. Simulations considering different combinations of well and barrier thicknesses, constrained to a total nine ML period, do not produce acceptable fits either. Using similar x-ray measurements Valcheva et al. have measured the period and well/barrier thickness of GaN/AIN SPSL grown by metal organic chemical vapor deposition on sapphire substrates. ${ }^{18}$ They reported $0.73 \mathrm{~nm}$ well and $2.28 \mathrm{~nm}$ barrier thicknesses that likewise do not correspond to integer multiples of $\mathrm{GaN}$ and AlN ML thickness, respectively. The difference between simulated and measured angular position of -1 satellite peak in Ref. 18 , which is $\sim 0.07^{\circ}$, is very close to the value of $0.068^{\circ}$ obtained for our sample discussed here.

Possible explanations for the observed deviations of

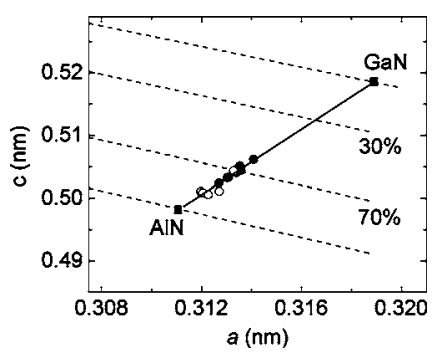

FIG. 3. The relationship between the $a$ and $c$ parameters of AlGaN alloys with varying the Al composition. The solid line is Vegard's law for relaxed material. The filled circles are for the SPSLs, the open circles are for random AlGaN alloys grown on sapphire, and the filled squares are for bulk GaN and AlN. Dashed lines illustrate the effect of biaxial strain for alloys of differing $\mathrm{Al}$ composition (\%).

measured well and barrier thicknesses from their expected integer ML multiples include stacking faults (SFs), inversion domain boundaries (IDBs), strain in the SPSL, composition fluctuations in the well and barrier, formation of interfacial layers, and interface roughness. Note that the interface roughness is usually attributed to two main effects: growth mode [2D or three dimensional (3D)] Refs. 17 and 18 and high density of TDs in III-nitrides. ${ }^{19-21}$ We now discuss each of these factors.

Heying et al. have shown that the mixed dislocations can split into partial dislocations and stacking faults lying along the [1100] direction. ${ }^{22}$ These stacking faults do not affect the symmetric $2 \theta-\omega$ scans. The inversion domain boundaries (IDBs) lying along the [0001] direction and in the (0001) plane were investigated in GaN based $p-n$ junctions. $^{23,24}$ It was shown that basal-IDBs, which do affect $2 \theta-\omega$ scans, were found only in $\mathrm{Mg}$-doped samples. ${ }^{23,24}$ Moreover these defects were not found in $\mathrm{Al}_{\mathrm{x}} \mathrm{Ga}_{1-x} \mathrm{~N}$ layers $(x \sim 0.08)$ (Ref. 25) suggesting differences in IDB formation in $\mathrm{GaN}$ and $\mathrm{Al}_{x} \mathrm{Ga}_{1-x} \mathrm{~N}$. Because our SPSLs contain $\mathrm{AlN}$ and $\mathrm{Al}_{x} \mathrm{Ga}_{1-x} \mathrm{~N}$ layers and do not contain $\mathrm{Mg}$ we neglect the influence of SFs and IDBs on the $2 \theta-\omega$ data.

Stress in III-N epitaxial materials generally arises from high lattice mismatches and differences in thermal expansion coefficients of these materials, including the substrates. The biaxial tensile stress perpendicular to the [0001] direction elongates the $a$ lattice constant, while decreasing the $c$ lattice parameter. Under compressive stress the situation is reversed. The relationship between the $a$ and $c$ parameters is expressed in terms of the elastic constants of the material. We employ the method suggested by Romano et al. ${ }^{26}$ to evaluate the $a$ and $c$ constants of AlGaN layers under stress. The solid line in Fig. 3 shows the relationship between the $a$ and $c$ parameters of relaxed $\mathrm{AlGaN}$ with varying $\mathrm{Al}$ composition. The family of dashed lines illustrates the effect of biaxial stress for different compositions. Intersections of the solid line with the dashed lines correspond to lattice constants of relaxed material at different compositions.

The location of the measured $a$ and $c$ values for an AlGaN layer in Fig. 3 shows the nature of stress in the layer. All the points lying above (below) the relaxation line are under compressive (tensile) biaxial stress. A line from a measured $(a, c)$ coordinate drawn parallel to the neighboring dashed lines will intersect the solid line to give the relaxed 


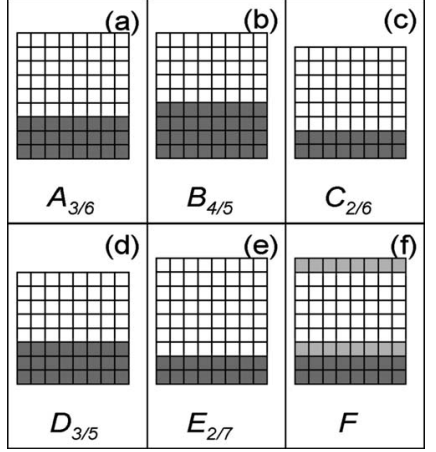

FIG. 4. Schematic cross-section depicting the model used to simulate SPSLs. [(a)-(e)] Abrupt interfaces; well/barrier thicknesses are three MLs/ six MLs (3/6), 4/5, 2/6,3/5, and 2/7, respectively. (f) Formation of ML thick interfacial layer (light grey color) between AlN barriers (white regions) and $\mathrm{AlGaN}$ wells (gray regions). Note that all well/barrier pairs can be randomly distributed in the vertical and lateral directions.

lattice parameters of the alloy layer, thus providing its composition. Based on the relaxed and strained lattice parameters, the stress in the layer, the average composition (determined using Vegard's law), and the degree of relaxation can be evaluated. The filled circles in Fig. 3 are the experimental values of the lattice parameters measured for our SPSLs. Also shown (open circles) are our experimental values of lattice parameters for random $\mathrm{Al}_{b} \mathrm{Ga}_{1-b} \mathrm{~N}, 0.6<b<0.9$, which are alloys grown on sapphire under conditions similar to those used for SPSLs and bulk GaN and AlN (filled squares) all measured using the same experimental setup. It is clear that our SPSLs and random alloy layers are relaxed. We conclude that stress cannot account for the observed discrepancies in the expected and measured values of SPSL thickness parameters.

Fluctuations in the well and barrier compositions do not adequately describe the differences between observed and expected well and barrier thicknesses. For example, a $1 \%$ change in the $\mathrm{Al}$ content in the well material corresponds to a change of $0.002 \mathrm{~nm}$ in the well thickness. In the epitaxial growth of alloy layers the composition variations are below $1 \%$. The expected variation in the well or barrier thickness is thus much smaller than the observed discrepancies in our SPSL layer thicknesses, suggesting that this effect may be ignored.

Ideal SPSL structures with no significant composition fluctuations are depicted in Figs. 4(a)-4(e). Each of these panels illustrates a well/barrier period composed of abrupt interfaces. Panels (a), (b), and (e) have period nine MLs, while panels (c) and (d) have eight ML period. Duplicating any of these along the growth and lateral directions will result in an ideal SPSL structure. For the case described here, Fig. 4(a) depicts the expected ideal well/barrier arrangement corresponding to three ML well and six ML barrier, denoted $3 / 6$ in Fig. 4. The same shorthand notation is used in each panel and in our later discussion. Combinations of these SPSL components may be used to address interface roughness when it occurs at the ML scale.

Another potential cause of discrepancy between observed and ideal layer thicknesses is the formation of interfacial layers with compositions intermediate to those of the
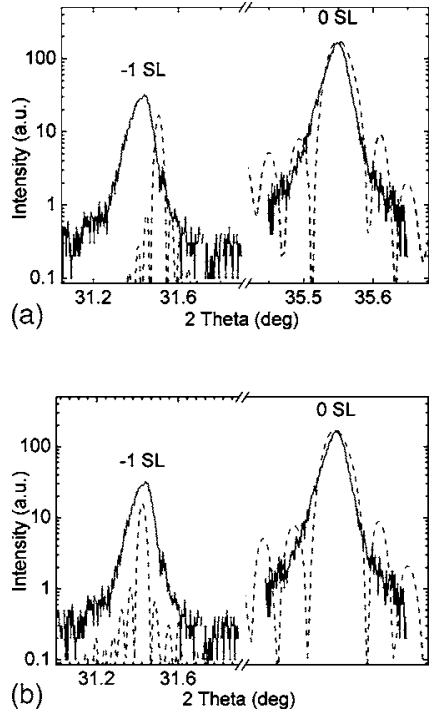

FIG. 5. X-ray data and simulations. (a) "Interfacial layer" model. (b) Rough interfaces.

well and barrier layers. This scenario is illustrated in Fig. 4(f). Adelmann et al. previously reported the formation of strained interfacial layers in plasma-assisted MBE growth of $\mathrm{AlN} / \mathrm{GaN} / \mathrm{AlN}$ single quantum well (QW) structures. $^{27}$ They found that AlGaN interfacial layers cannot be formed by interdiffusion of $\mathrm{Ga}$ and $\mathrm{Al}$ at growth temperature of $730{ }^{\circ} \mathrm{C}$ under group-III-rich conditions. Although there is a preferential incorporation of $\mathrm{Al}$, even in the presence of $\mathrm{Ga}$, they found that the width of this interface is $\sim$ one ML when $\mathrm{GaN}$ is grown on AlN and $\sim$ two MLs when AlN is grown on GaN. Simulations taking into account the formation of strained interfacial layers in our SPSLs do not fit our x-ray data. There are a few differences between our growth conditions and those reported in Ref. 27. All our SPSLs were grown under $\mathrm{N}$-rich conditions at $780{ }^{\circ} \mathrm{C}$. N-rich conditions exclude the preferential incorporation of $\mathrm{Al}$ with respect to $\mathrm{Ga}$ when the $\mathrm{Ga}$ and $\mathrm{Al}$ effusion cell shutters are switching from the barrier to well growth, and vice versa. In addition, the $\sim 1 \mathrm{~s}$ required to open and close the shutters and $\sim 3 \mathrm{~s}$ required to stabilize $\mathrm{Ga}$ and $\mathrm{Al}$ fluxes result in varying flux of these elements at the growth surface. Note, the well and barrier growth times range from 5 to $8 \mathrm{~s}$ and from 20 to $30 \mathrm{~s}$, respectively. High growth temperature and long total growth time $(\sim 3 \mathrm{~h})$ suggest that we may not exclude $\mathrm{Al}$ and $\mathrm{Ga}$ interdiffusion.

The interdiffusion, shutter delays of the MBE system, and flux transients may result in the formation of interfaces that are not abrupt at the monolayer level, as illustrated in Fig. 4(f). Note that the interdiffusion should have a similar result on the composition at both the well/barrier and barrier/ well interfaces, while the flux transient should yield Ga-rich barrier/well and Al-rich well/barrier interfaces. The best simulation to the zeroth peak is obtained assuming ML interface composition averaging $z=\left(z_{\mathrm{bw}}+z_{\mathrm{wb}}\right) / 2 \sim 0.47$ for both the barrier/well and well/barrier interfaces. We refer to this approach as the "interfacial layer" model. Figure 5(a) compares the experimental results with simulated interfacial layer curves. Although the satellite position is very close to 
the experimental curve, within $\sim 0.068^{\circ}$, it corresponds to a difference in the period of $\sim 0.038 \mathrm{~nm}$. This difference cannot be attributed to the residual stress and/or composition fluctuations in SPSL described above. Thus, while the formation of interface layers with intermediate composition describes the zeroth order diffraction, this model alone does not adequately interpret the satellite position.

We now consider the effects of interface roughness, resulting from ML steps and threading dislocations at the interfaces. Under our 2D growth conditions for both thin and thick layers, the $\mathrm{Al}_{0.08} \mathrm{Ga}_{0.92} \mathrm{~N}$ composition used in the wells produces exceptionally smooth surface characteristic of the step-flow mode. In contrast, $\mathrm{AlN}$ grown at $780{ }^{\circ} \mathrm{C}$ is formed in the $3 \mathrm{D}$ mode for layers of $5 \mathrm{~nm}$ or thicker. Although this is thicker than our barriers $(\leqslant 1.5 \mathrm{~nm})$, it suggests the possibility that the AlN layers might be rougher than the well layers. Even two or more ML steps are possible in this case. However, our cross-sectional transmission electron microscopy (TEM) examination ${ }^{1}$ of SPSLs did not reveal ML steps, showing instead smooth and abrupt well-barrier interfaces. Valcheva et al. reported similar TEM results for GaN/AIN SPSL. ${ }^{17}$ They concluded that the internal strains in coherently grown SPSL can accommodate a lattice mismatch rather than formation of misfit dislocations. ${ }^{17}$ This does not rule out ML steps, however, since TEM studies address a short lateral scale of $\sim 500 \mathrm{~nm}$. Our AFM measurements ${ }^{10}$ yield rms surface roughness of $\sim 1 \mathrm{~nm}$ ( $\sim$ four MLs), in the large area of $20 \times 20 \mu \mathrm{m}^{2}$ scan, indicating variations in the period may be present within the SPSL stack. These height variations observed at the surface are cumulative for the entire $\sim 0.8 \mu \mathrm{m}$ stack and do not directly correspond to interface roughness. However, these studies indicate that ideal 2D SPSL structures are not obtained, suggesting that a more accurate model should include alternatives to the ideal well/ barrier pair such as weighted combinations of the ideal structures depicted in Figs. 4(a)-4(e). For example, the step-flow growth mode can be modeled combining Fig. 4 panels (a) and (b) or (b) and (c), while 3D growth may be described using panels (b) and (e) or (d) and (e).

Threading dislocations also have an impact on ML-scale interface roughness. It is well known that TDs (screw, edge, and mixed) are the dominant defects in III-N structures ${ }^{19,20,28-31}$ leading to lattice distortions. Screw dislocations distort all $(h k l)$ planes with $l \neq 0$, while edge dislocations distort $(h k l)$ planes with $h, k \neq 0 .{ }^{20}$ Thus only screw dislocations affect the rocking curves of the symmetric (0002) reflections while edge dislocations affect the rocking curves of asymmetric (10 $\overline{1} 0)$ planes. Although the mixed dislocation cannot be directly detected by XRD, their screw and edge components contribute to the broadening of symmetric and asymmetric reflections, respectively. ${ }^{13}$ Using approaches described above ${ }^{14,15}$ the screw and edge dislocation densities in our SPSLs were determined to range between $(1-4)$ $\times 10^{9}$ and $(0.9-4) \times 10^{10} \mathrm{~cm}^{-2}$, respectively. For the sample detailed here we determined screw and edge dislocation densities of $2 \times 10^{9} \mathrm{~cm}^{-2}\left(\mathrm{FWHM}_{0002}=1020 \mathrm{arc} \mathrm{sec}\right)$ and 2 $\times 10^{10} \mathrm{~cm}^{-2}\left(\mathrm{FWHM}_{1010}=1870\right.$ arc sec $)$, respectively. Although the edge dislocation densities in our SPSLs are very high they do not change the lattice spacing of the (0002) planes. ${ }^{13}$ Since the superlattice parameters are based on the position of SPSL peaks of $2 \theta-\omega$ scans, which are governed only by the lattice spacing, we conclude that the high edge dislocation density does not affect our calculations of the SPSL parameters.

Screw dislocations, spiral in nature and propagating perpendicular to the substrate surface, result in surface roughening. ${ }^{32}$ For the samples grown on (0001) sapphire the Burgers vector $\mathbf{b}$ of screw dislocation is $\mathbf{b}=\mathbf{c}=$ [0001], where $\mathbf{c}$ is the unit vector of hexagonal III-nitride unit cell. ${ }^{33}$ Thus, these dislocations can be modeled by simulating well-barrier pair combinations having thicknesses which deviate from the nominal values by integer multiples of ML in different regions of the sample. ${ }^{34}$ We assume differently weighted wellbarrier pairs which are randomly distributed along the [0001] direction. We have considered pairs which alter the well or barrier thickness by one ML and found that well/barrier combinations $3 / 6,4 / 5,2 / 6,3 / 5$, and $2 / 7 \mathrm{ML}$ adequately describe the x-ray data. We refer to these pairs as $A, B, C, D$, and $E$, respectively, corresponding to Figs. 4(a)-4(e). The data and simulations are shown in Fig. 5(b). The best fit was obtained using $A, B, C, D$, and $E$ pairs correspondingly weighted according to $58 \%, 24 \%, 14 \%, 2 \%$, and $2 \%$. The prominent weighting of $A$ pairs, $\sim 58 \%$, is expected from the nominal $3 / 6 \mathrm{ML}$ thickness from growth for this sample. The net contributions of interface roughing by one ML deviation in either well or barrier, $B$ plus $C$, is around $38 \%$. The contributions of interface roughening by two ML deviations from the nominal $3 / 6, D$ plus $E$, is small. However, neglecting the contributions of $D$ and $E$ in the simulations result in a noticeable shift in the zeroth and satellite peak positions. We conclude from this analysis that the one ML interface roughening from the step-flow growth mode and the presence of TDs plays an important role in the angular position of satellite diffraction peaks. This roughening must be taken into account in understanding the observed discrepancies in the expected and measured values of SPSL thickness parameters.

As mentioned above, the "interfacial layer" model fits well the angular position of zeroth SL peak but does not adequately describe the position of satellite peaks. However, when adding the effects of interface roughening we obtain good agreement for both zeroth and the satellites. For completeness, we have also considered the possibility of composition changes at the SPSL interfaces due to shutter delays of the MBE system and flux transients. The model builds upon simulations describing TDs and step-flow growth mode, described in the previous paragraphs, plus the interfacial layer modeled as depicted in Fig. 4(f). All well/barrier combinations are likewise differently weighted and randomly distributed along [0001] direction. This approach also results in consistent agreement between simulation and data for both diffraction peaks. The best fit was obtained using $48 \%, 20 \%$, $12 \%, 2 \%$, and $2 \%$ weight proportion for $A, B, C, D$, and $E$, respectively, plus $16 \%$ weighting for the interfacial layer. Again, the 3/6 combination is dominant. The net contributions of interface roughing by one ML deviation in either well or barrier, $B$ plus $C$, is now around $32 \%$ and the two ML combinations have the same small weight as above. The av- 
erage interfacial layer, with $16 \%$ weight, has composition for this SPSL of $z=\left(z_{\mathrm{bw}}+z_{\mathrm{wb}}\right) / 2=0.40$. Note that the value of $z=0.40$ differs from $z=0.47$ obtained above from simulating the interfacial layer effect alone. The difference is reasonable since we now combine the two effects of interface roughness and interfacial layers to obtain the best simulation of our data. Since the addition of these interfacial layers does not significantly improve the agreement between simulations and experiment, we conclude that interface roughness due to the step-flow growth mode and presence of screw dislocations is sufficient to interpret our $\mathrm{x}$-ray data.

\section{SUMMARY}

HRXRD measurements of AlGaN SPSLs, with periods ranging from 1.27 to $2.53 \mathrm{~nm}$, reveal large deviations between well and barrier thicknesses and what is expected from integer multiples of MLs. To obtain reliable structural information on SPSLs, it is important to model both the zeroth and either of the \pm 1 satellite peaks. We conclude that interface roughness, due to screw dislocations and step-flow growth mode, plays a dominant role in the satellite peak positions. Data are adequately described considering contributions from the expected well/barrier thickness combination, based on the growth conditions, along with one ML and two MLs randomly distributed deviations from this expected value. When the role of interfacial layer formation is also considered, the nominal composition and ML deviations still play the dominant roles.

\section{ACKNOWLEDGMENTS}

We acknowledge support from NSF (ECS-0304224 and ECS-0609416), THECB-ARP-003644-0014-2006, and the J. F Maddox Foundation. We also thank G. Kipshidze and B. Borisov for growth of samples.

${ }^{1}$ S. A. Nikishin, M. Holtz, and H. Temkin, Jpn. J. Appl. Phys., Part 1 44, 7221 (2005), and references therein.

${ }^{2}$ Z. Sitar, M. J. Paisley, B. Yan, J. Ruan, W. J. Choyke, and R. F. Davis, J. Vac. Sci. Technol. B 8, 316 (1990).

${ }^{3}$ M. A. Khan, J. N. Kuznia, D. T. Olson, T. George, and W. T. Pike, Appl. Phys. Lett. 63, 3470 (1993).

${ }^{4}$ H. Hirayama, A. Kinoshita, M. Ainoya, A. Hirata, and Y. Aoyagi, Phys. Status Solidi A 188, 83 (2001).

${ }^{5}$ G. Kipshidze, V. Kuryatkov, K. Zhu, B. Borisov, M. Holtz, S. Nikishin, and H. Temkin, J. Appl. Phys. 93, 1363 (2003).

${ }^{6} \mathrm{H}$. Temkin, in Advanced Semiconductor and Organic Nano-techniquesPart-I, edited by H. Morkoç (Academic, San Diego, 2003), and references therein.
${ }^{7}$ H. Hirayama, J. Appl. Phys. 97, 091101 (2005), and references therein. ${ }^{8}$ G. Kipshidze, V. Kuryatkov, B. Borisov, S. Nikishin, M. Holtz, S. N. G. Chu, and H. Temkin, Phys. Status Solidi A 192, 286 (2002).

${ }^{9}$ V. Kuryatkov, A. Chandolu, B. Borisov, G. Kipshidze, H. Temkin, K. Zhu, S. Nikishin, and M. Holtz, Appl. Phys. Lett. 82, 1323 (2003).

${ }^{10}$ S. A. Nikishin, B. A. Borisov, A. Chandolu, V. V. Kuryatkov, H. Temkin, M. Holtz, E. N. Mokhov, Yu. Makarov, and H. Helava, Appl. Phys. Lett. 85, 4355 (2004).

${ }^{11}$ M. Holtz, I. Ahmad, V. V. Kuryatkov, B. A. Borisov, G. D. Kipshidze, A. Chandolu, S. A. Nikishin, and H. Temkin, Mater. Res. Soc. Symp. Proc. 798, Y1.9.1 (2004).

${ }^{12}$ P. F. Fewster, N. L. Andrew, and C. T. Foxon, J. Cryst. Growth 230, 398 (2001).

${ }^{13}$ B. Heying, X. H. Wu, S. Keller, Y. Li, D. Kapolnek, B. P. Keller, S. P. DenBaars, and J. S. Speck, Appl. Phys. Lett. 68, 643 (1996).

${ }^{14}$ V. M. Kaganer, O. Brandt, A. Trampert, and K. H. Ploog, Phys. Rev. B 72, 045423 (2005).

${ }^{15}$ H. Heinke, V. Kirchner, S. Einfeldt, and D. Homme, Phys. Status Solidi A 176, 391 (1999).

${ }^{16}$ H. Heinke, V. Kirchner, S. Einfeldt, and D. Homme, Appl. Phys. Lett. 77, 2145 (2000).

${ }^{17}$ D. Hull and D. J. Bacon, Introduction to Dislocations (Pergamon, Oxford, 1984).

${ }^{18}$ E. Valcheva, T. Paskova, G. Z. Radnoczi, L. Hultman, B. Monemar, H. Amano, and I. Akasaki, Physica B 340-342, 1129 (2003).

${ }^{19}$ K. Kusakabe, A. Kikuchi, and K. Kishino, Mater. Res. Soc. Symp. Proc. 639, G3.33.1 (2001).

${ }^{20}$ V. Srikant, J. S. Speck, and D. R. Clarke, J. Appl. Phys. 82, 4286 (1997).

${ }^{21}$ R. Chierchia, T. Böttcher, H. Heinke, S. Einfeldt, S. Figge, and D. Hommel, J. Appl. Phys. 93, 8918 (2003).

${ }^{22}$ B. Heying, E. J. Tarsa, C. R. Elsass, P. Fini, S. P. DenBaars, and J. S. Speck, J. Appl. Phys. 85, 6470 (1999).

${ }^{23}$ I. Arslan, A. Bleloch, E. A. Stach, and N. D. Browning, Phys. Rev. Lett. 94, 025504 (2005).

${ }^{24}$ V. Ramachandran, R. M. Feenstra, W. L. Sarney, L. Salamanca-Riba, J. E. Northrup, L. T. Romano, and D. W. Greve, Appl. Phys. Lett. 75, 808 (1999).

${ }^{25} \mathrm{Ph}$. Komninou, Th. Kehagias, J. Kioseoglou, G. P. Dimitrakopulos, A. Sampath, T. D. Moustakas, G. Nouet, and Th. Karakostas, J. Phys.: Condens. Matter 14, 13277 (2002).

${ }^{26}$ L. T. Romano, D. Hofstetter, M. D. McCluskey, D. P. Bour, and M. Kneissl, Appl. Phys. Lett. 73, 2706 (1998).

${ }^{27}$ O. Briot, S. Ruffenach-Clur, M. Moret, and R. L. Aulombard, Mater. Res. Soc. Symp. Proc. 639, G4.40.1 (2001).

${ }^{28}$ C. Adelmann, E. Sarigiannidou, D. Jalabert, Y. Hori, J. L. Rouvière, B. Daudin, S. Fanget, C. Bru-Chevallier, T. Shibata, and M. Tanaka, Appl. Phys. Lett. 82, 4154 (2003).

${ }^{29}$ D. Cherns, Y. Q. Wang, R. Liu, and F. A. Ponce, Appl. Phys. Lett. 81, 4541 (2002).

${ }^{30}$ Z. Liliental-Weber, D. Zakharov, J. Jasinski, M. A. O'Keefe, and H. Morkoç, Microsc. Microanal. 10, 47 (2004).

${ }^{31}$ F. A. Ponce, D. Cherns, W. T. Young, and J. W. Steeds, Appl. Phys. Lett. 69, 770 (1996).

${ }^{32}$ J. E. Northrup, Appl. Phys. Lett. 78, 2288 (2001).

${ }^{33}$ T. Metzger, R. Höpler, E. Born, O. Ambacher, M. Stutzmann, R. Stömmer, M. Schuster, H. Göbel, S. Christiansen, M. Albrecht, and H. P. Strunk, Philos. Mag. A 77, 1013 (1998).

${ }^{34}$ S. B. Hendricks, Am. Mineral. 40, 139 (1955). 